\title{
Electric birefringence study of the dielectric properties of anisotropic and electrically charged latex particles
}

\author{
V. DEGIORGIO, F. MANTEGAZZA, M. GIARDINI, R. PIAZZA and T. BELLINI \\ Dipartimento di Elettronica, Sezione di Fisica Applicata, Universitd di Pavia, 27100 Pavia, Italy
}

Static and dynamic electric birefringence measurements are performed on aqueous dispersions of electrically charged fluorinated latex particles having an almost spherical shape. The Kerr constant is nonzero because of the intrinsic and form anisotropy of the particles, and is considerably enhanced at low frequencies by a contribution due to the cloud of dissociated counterions. We discuss in detail this effect which shows a non-Lorentzian frequency dispersion, and an amplitude strongly dependent on the particle concentration and on the ionic strength. The data are interpreted by making a connection with the theory of the dielectric constant of polyelectrolyte solutions.

La biréfringence électrique de dispersions acqueuses de polymères colloidaux fluorés, de forme presque-sphérique et avec une charge électrique, a été étudiée par des mesures statiques et dynamiques. La constante de Kerr est différente de zéro à cause de l'anisotropie intrinsèque et de l'anisotropie de forme des particules, et est considérablement augmentée à basses fréquences par un effet lié à la présence du nuage de contre-ions. Cet effet présente une dispersion en fréquence non-Lorentzienne, et une amplitude qui dépend fortement de la concentration en particules et de la force ionique. Les résultats sont interprétés en liaison avec la théorie de la constante diélectrique des solutions de polyélectrolytes. 


\section{Introduction.}

The frequency-dependent dielectric properties of polyelectrolyte solutions have been investigated by many authors on a variety of systems, such as colloidal dispersions or solutions of proteins, nucleic acids, synthetic polyelectrolytes and micelles. However, because of the complexity of the problem, the understanding of the observed phenomena is still rather limited [1]. The presence of mobile charges makes polyelectrolyte solutions strongly electrically active: both the dielectric constant [2-6] and the Kerr constant [7-19] of the solution may become quite large even at low polyelectrolyte concentrations. Formulation of an adequate theory for the dielectric constant and the electric conductivity of these systems requires treatment of the ion transport processes in the solvent, in the ion atmosphere surrounding the particle, and near the surface of the suspended particle. Transient electric birefringence (TEB) studies can give important information on the dielectric properties of the solution, mainly because they allow to easily separate contributions due to different physical mechanisms.

TEB experiments have been performed by various authors with many different polyelectrolyte solutions, including rigid particles with simple geometrical shapes [7-10], flexible polymer chains [1117], and ionic micellar solutions [18,19]. In the case of Tobacco Mosaic Virus (TMV) solutions, the available data [6] indicate the presence of two distinct dielectric relaxations, one with a cut-off frequency of a few hundred $\mathrm{Hz}$ and the other with a cut-off frequency around $30 \mathrm{kHz}$. Effects similar to those observed with TMV solutions have also been found in TEB experiments on polyelectrolytes, like DNA [11] and sodium polystyrene sulfonate (NaPSS) [12-17]. In the case of flexible polyelectrolytes data interpretation is more difficult than for rigid particles because they present a statistical configuration which changes when some experimental parameters, like the ionic strength or the polyelectrolyte concentration, are varied. Even more complex phenomena are observed when the system is not in the dilute regime: for instance, Hoffmann and coworkers $[17,18]$ describe the so-called electric birefringence anomaly which consists in a change of sign of the birefringence measured as a function of time when the amplitude of the applied field is kept constant for a sufficiently long time interval.

In order to get a clearer picture, it is helpful to study first a simple system consisting in a dilute suspension of monodisperse rigid macroions. To this end, we have undertaken a series of TEB measurements on dilute aqueous dispersions of fluorinated latex particles which are made from a polytetrafluoroethylene copolymer (PFA). The properties of these particles have been recently investigated in detail by using static and dynamic light scattering [20]. It was found that the PFA particles are rather monodisperse and quasi spherical, have an index of refraction close to that of water and possess an intrinsic optical anisotropy. They are, therefore, a very interesting model system for a TEB study of the dielectric properties of polyelectrolytes. By measuring the response to the applied electric field both in the time and frequency domain, we were able to distinguish three distinct contributions to the specific Kerr constant B/ $\Phi$, B being the Kerr constant of the dispersion and $\Phi$ the particle volume 
fraction. The first contribution has an electronic origin and does not depend on the frequency of the applied field, the second appears only at low frequencies and is due to the presence of charged sites and bound (but mobile) counterions on the particle surface, the third is dependent on the particle volume fraction and on the concentration of added salt and is connected with the dynamics of the cloud of nonassociated counterions and with the interactions among clouds belonging to different particles. In our first paper on this subject [10] we have thoroughly discussed the first two contributions, in this article we focus our attention on the third one. In particular, we show that the amplitude of the third contribution is proportional to the volume fraction of particles and is a strongly decreasing function of the ionic strength. Its frequency dispersion has a non-Lorentzian shape which is independent from both salt and particle concentration. The results are explained by using a mean-field phenomenological argument which shows the approximate proportionality between $B / \Phi$ and the dielectric constant of the dispersion. In fact, the measured frequency dispersion of $B / \Phi$ fits very well the theoretical prediction for the frequency dependence of the dielectric constant of a dispersion of spherical polyelectrolytes [21-23].

\section{Properties of the colloidal particles.}

We have used an aqueous dispersion of nearly monodisperse latex particles of a alkoxyvinylether tetrafluoroethylene copolymer (PFA, commercially known as Hyflon) obtained from Montefluos S.p.A., Spinetta Marengo (Italy). The particles have an average index of refraction, $n_{p}=1.352$, close to that of water, and present an appreciable intrinsic optical anisotropy, which derives from their partially crystalline internal structure [20]. The measured anisotropy of the refractive index is: $\delta n_{i n t}=n_{1}-n_{2}=$ 0.0051 , where the index 1 refers to the direction parallel to the simmetry axis of the particle, and the index 2 to any direction perpendicular to the simmetry axis. The shape of the particles is close to spherical, but not exactly spherical. Modelling the particle as a prolate ellipsoid with semiaxes a,b,b, we obtained a fairly good agreement with the measured value of the Kerr constant taking for the ratio between the semiaxes the value $a / b=1.025$ [10]. The fact that such a small ellipticity can be rather easily measured by electric birefringence should not be surprising if we note that electric birefringence is a differential technique with regard to nonsphericity.

The TEB results obtained with the reversing pulse technique have been interpreted by assuming that the particles possess a fluctuating electric dipole [10]. The origin of the fluctuating electric dipole in a charged particle can be related to statistical fluctuations of the charge distribution on the surface, as it was proposed some time ago by Kirkwood and Shumaker to account for the dielectric response of globular proteins [24]. If the center of charge does not coincide for a given particle with the center of mass, an applied electric field will generate a torque tending to align along its direction the center of charge displacement vector, and this is equivalent to the effect of an electric dipole moment. Since the position of bound counterions is fluctuating with time, the random dipole moment of the particle presents 
temporal fluctuations with zero average. The measured correlation time is about $1 \mathrm{~ms}$.

\section{Experimental results}

The experiment was performed on particles with radius $R=90 \mathrm{~nm}$ at volume fractions $\Phi$ ranging from $10^{-3}$ to $2.5 \times 10^{-2}$. The original latex $(\Phi \approx 20 \%)$ was dyalized for some weeks until its conductivity reached a stable value of about $100 \mu \mathrm{S} / \mathrm{cm}$, and then diluted with a small quantity of nonionic surfactant added to prevent any coagulation. Some measurements were performed by adding $\mathrm{NaCl}$ to the solution in order to investigate the dependence of the response on the ionic strength.

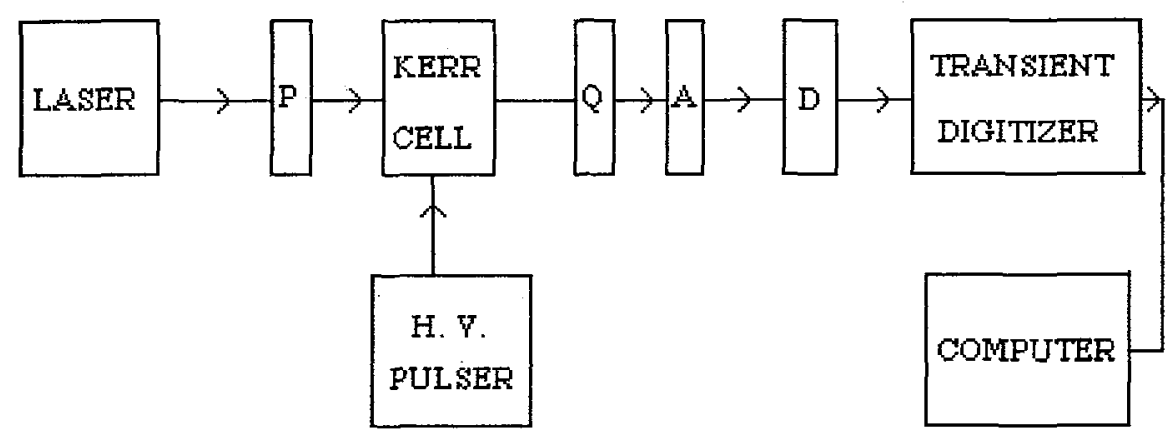

Fig. 1. Scheme of the experimental set-up. $P$ and $A$ are polarizers, $Q$ is a quarter-wave plate, $D$ is the photodetector.

A general description of our optical and detection apparatus can be found in Ref.25. The electric birefringence experiment [26-29] essentially consists in applying a rectangular voltage pulse to the electrodes of the Kerr cell with a pulse duration long enough to reach a stationary value of the induced anisotropy, and in observing the induced birefringence pulse by using a laser beam and two almost crossed polarizer. A scheme of the set-up is shown in Fig.1. For the measurements that are discussed in this paper the applied electric field consisted of a sine-wave pulse with zero average and frequency variable between $100 \mathrm{~Hz}$ and $2 \mathrm{MHz}$. The pulse was obtained by gating a function generator and amplifying the output with a Krohn-Hite 7500 power amplifer. The pulse duration was in the range 1-10 ms. Sine-wave pulses with variable frequency and zero time average allow, at the same time, to avoid steady-state electric currents in the cell and to single out the contribution of distinct polarization mechanisms at different frequencies. The birefringence signal is obtained by putting the Kerr cell between two polarizers (with the insertion of a quarter-wave plate between cell and analyzer) and by sending a laser beam through the optical system. Signal averaging over many pulses was performed by a 
Data Precision DATA 6100 digital oscilloscope. All the reported results were obtained in the Kerr regime where the induced birefringence $\Delta \mathrm{n}$ is proportional to the square of the electric field $\mathrm{E}$. The Kerr constant is defined as: $B=\Delta \mathrm{n} /\left(\lambda E^{2}\right)$, where $\lambda$ is the wavelength of the laser beam. In our experiment, $\lambda=0.633$ $\mu \mathrm{m}$.

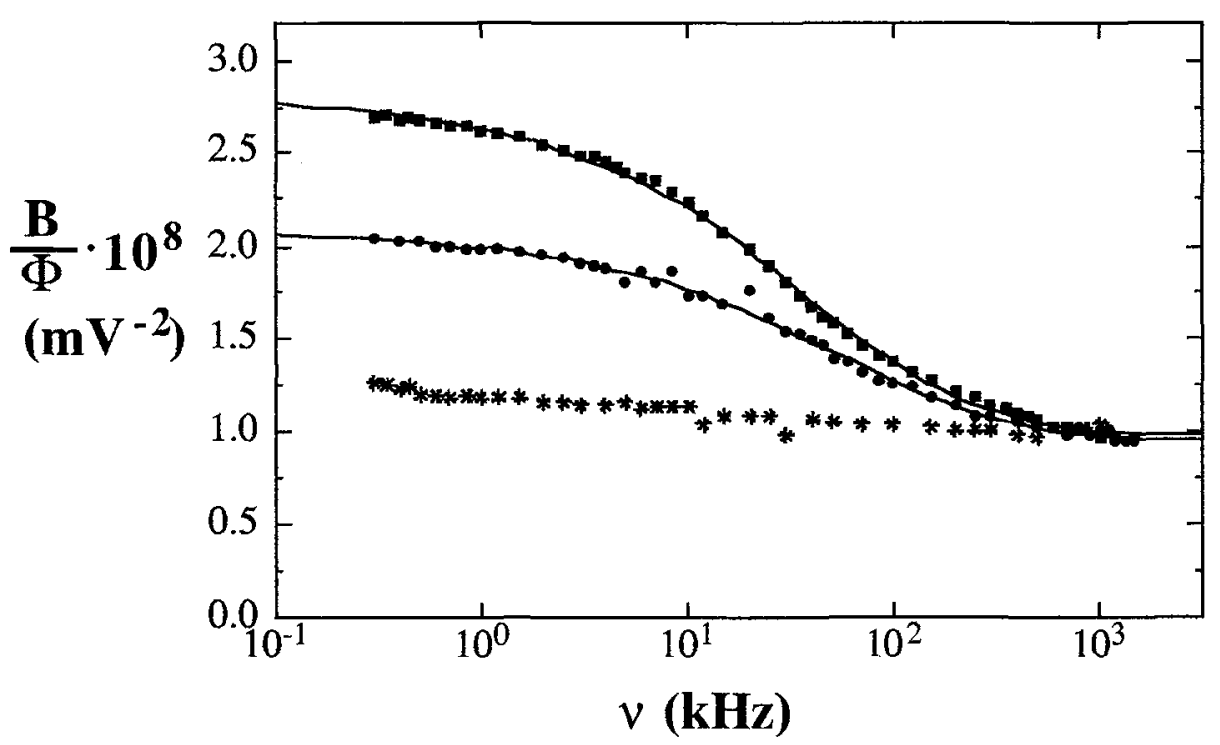

Figure 2. The specific Kerr constant measured as a function of the frequency of the electric field, at $\mathrm{c}_{\mathrm{s}}=0.2 \mathrm{mM}$, for three distinct volume fractions: $\left(^{*}\right), \Phi=0.1 \% ;(\bullet), \Phi=1 \% ;(\square), \Phi=$ $2.5 \%$. The full lines represent a fit with Eq.6.

The electric birefringence response to a sinusoidal field consists of a d.c. component plus a sinusoidal component oscillating at twice the frequency $v$ of the exciting signal [8]. The amplitude of the sinusoidal component is a decreasing function of $v$, with a cut-off frequency around $\left(6 \pi \tau_{r}\right)^{-1}$.We call $\tau_{r}$ the rotational diffusion time of the particle, $\tau_{\mathrm{r}}=4 \pi \eta \mathrm{R}^{3} /\left(3 \mathrm{k}_{\mathrm{B}} \mathrm{T}\right)$, where $\eta$ is the viscosity of the solvent, $k_{B}$ the Boltzmann constant and $T$ the absolute temperature. After the applied electric field is off, the induced birefringence decays exponentially, with a decay constant $\tau_{\mathrm{r}}$. We obtained from our data $\tau_{\mathrm{r}}=(605 \pm 20)$ $\mu \mathrm{s}$, in good agreement with the theoretical expression.

We have studied dispersions with concentrations of added $\mathrm{NaCl}, \mathrm{c}_{\mathrm{s}}$, in the range $0-5 \mathrm{mM}$. Dispersions without added salt have an electric conductivity in the range of few $\mu \mathrm{S} / \mathrm{cm}$ which is much larger than that expected from the particle counterions only and is mainly due to the carbon dioxide dissolved in water. 
In all cases, the decay of the induced birefringence pulse is found to be exponential with a decay constant coinciding with $\tau_{\mathrm{r}}$. This means that, in all the investigated situations, electrostatic interactions have little effect on the rotational Brownian motion of the individual particle.

It should be recalled that, when the frequency of the applied field is very low (say, below $200 \mathrm{~Hz}$ ), it is possible to notice an additional contribution to $B / \Phi$ which comes from the fluctuating dipole moment [10]. The low frequency data discussed in this article are obtained at frequencies large enough to exclude contributions coming from the fluctuating electric dipole.

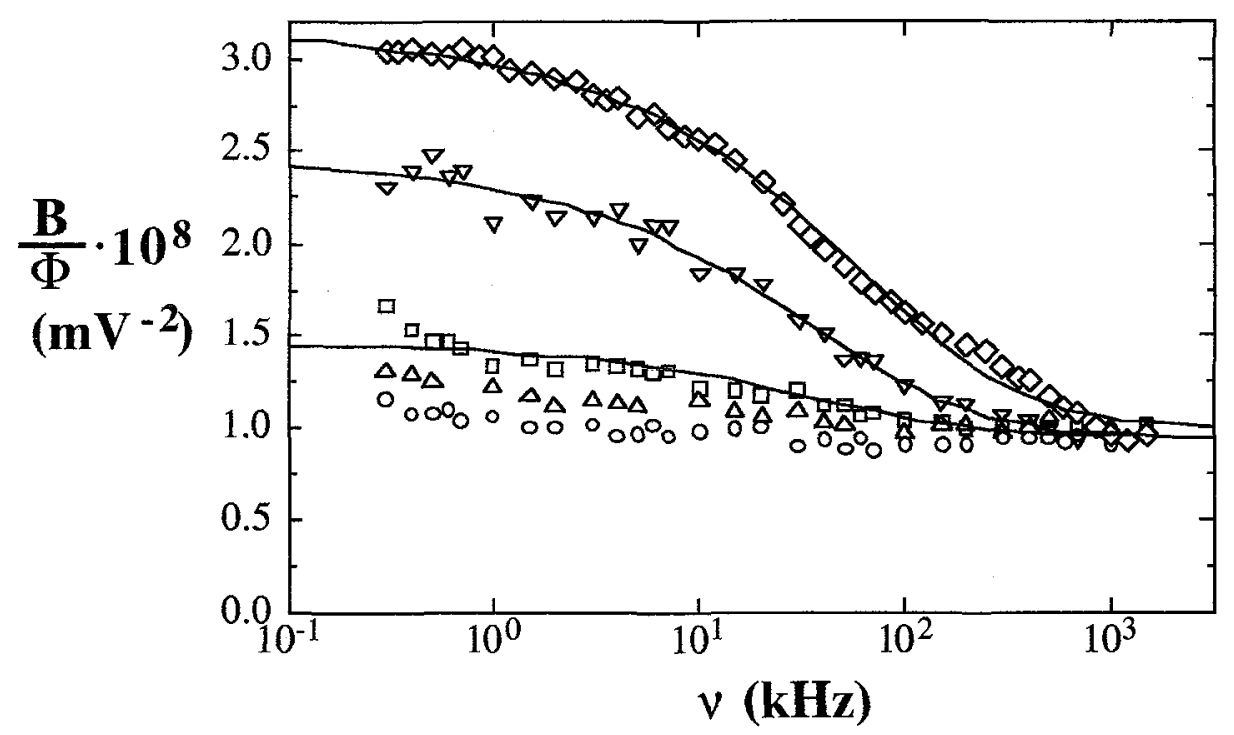

Fig. 3. The specific Kerr constant measured as a function of the frequency of the electric field, at $\Phi=1.5 \%$, for five distinct concentrations of added $\mathrm{NaCl}:(\bigcirc), \mathrm{c}_{\mathrm{s}}=0 \mathrm{mM} ;(\nabla), \mathrm{c}_{\mathrm{s}}=0.2 \mathrm{mM}$;

$(\square), c_{s}=1 \mathrm{mM} ;(\Delta), c_{s}=3 \mathrm{mM} ;(\bigcirc), c_{\mathrm{s}}=5 \mathrm{mM}$. The full lines represent a fit with Eq.6.

We report in Fig. 2 the specific Kerr constant $B / \Phi$, derived from the steady-state d.c. component, as a function of the frequency $v$ of the exciting wave, at various volume fractions $\Phi$. All the data of Fig. 2 were obtained at $c_{\mathrm{s}}=0.2 \mathrm{mM}$. We note that the Kerr constant is positive. The amplitude of the frequency-dependent contribution to $B / \Phi$ grows with $\Phi$, leaving substantially unchanged the shape of the frequency response which presents a cut-off frequency around $30 \mathrm{kHz}$. As shown in Fig.3, the amplitude of such a contribution is a rapidly decreasing function of salt concentration, becoming negligible when $c_{\mathrm{s}}$ exceeds $5 \mathrm{mM}$. At $v \geq 1 \mathrm{MHz}, \mathrm{B} / \Phi$ becomes independent of both $\mathrm{c}_{\mathrm{s}}$ and $\Phi$, and takes the value $(0.90 \pm 0.05) \times 10^{-8} \mathrm{mV}^{-2}$. The ratio between the low and high frequency values of $B / \Phi$ was 
measured as a function of $\Phi$ for several salt concentrations: $B_{L F}$ is measured at $v=2.5 \mathrm{kHz}$, and $B_{H F}$ at $v=1 \mathrm{MHz}$. Some data are shown in Fig.4. The ratio increases linearly with $\Phi$ in the range of investigated volume fractions. By fitting with a linear function, $\mathrm{B}_{\mathrm{LF}} / \mathrm{B}_{\mathrm{HF}}=\mathrm{A}(1+\mathrm{k} \Phi)$, we have derived the slope $\mathrm{k}$ as a function of the salt concentration. The results are shown in Fig.5. The behavior of $\mathrm{k}$ can be approximately described as $\mathrm{k} \approx \mathrm{c}_{\mathrm{s}}{ }^{-} \beta$, with $\beta=0.7 \pm 0.1$. We have also reported in Fig.5 the values of $\mathrm{k}$ obtained in our previous experiment [9] with fluorinated rod-like polyelectrolyte particles having an axial ratio about 2 . We find that $k$, at fixed $c_{s}$, is larger for the rod-like particles by one order of magnitude, and that, for the rod-like particles, $\beta=1.0 \pm 0.1$.

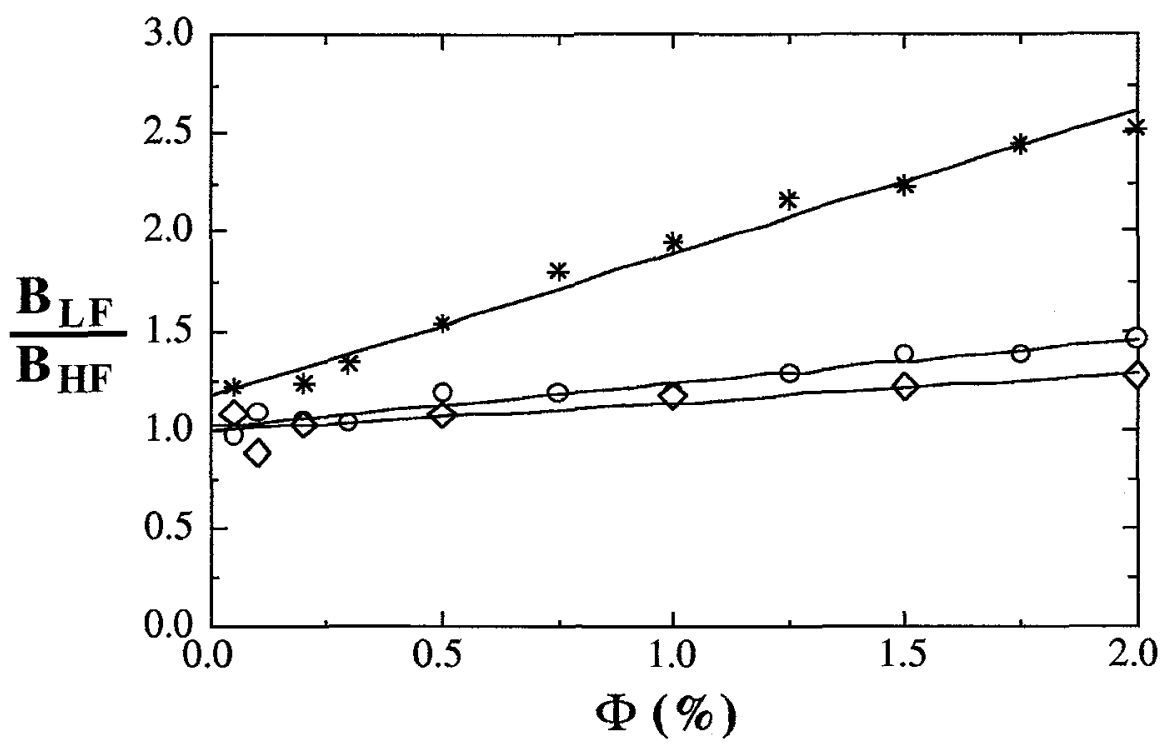

Fig. 4. The ratio $\mathrm{B}_{\mathrm{LF}} / \mathrm{B}_{\mathrm{HF}}$ plotted as a function of the volume fraction $\Phi:(*), c_{\mathrm{S}}=0.2 \mathrm{mM} ;(\varnothing), \mathrm{c}_{\mathrm{s}}=$ $1 \mathrm{mM} ;(\bigcirc), \mathrm{c}_{\mathrm{s}}=2 \mathrm{mM}$.

\section{Discussion.}

As shown in our previous paper [10], the Kerr constant of the colloidal dispersion is made of three contributions. The frequency-independent contribution, which is associated with an electronic polarization mechanism, contains information about the shape and the intrinsic anisotropy of the particle. The other two contributions are connected with the fact that the particle is a polyion. The standard view assumes that counterions can be separated in two distinct categories: condensed counterions which are 
constrained to stay close to the particle surface, and dissociated counterions which are spread over a distance of the order of the Debye-Hückel length. We have suggested that the contribution present at very low frequencies, which becomes evident with the reversing-pulse technique, comes from a fluctuating electric dipole originated from the dynamics of bound counterions [10]. The contribution which gives the frequency dispersion of $B / \Phi$ shown in Figs. 2 and 3 is instead connected with the existence of the cloud of dissociated counterions. As shown by the data of Fig.4, this latter effect is predominantly collective, that is, it becomes important when there is an appreciable overlap of the counterion clouds belonging to different particles.

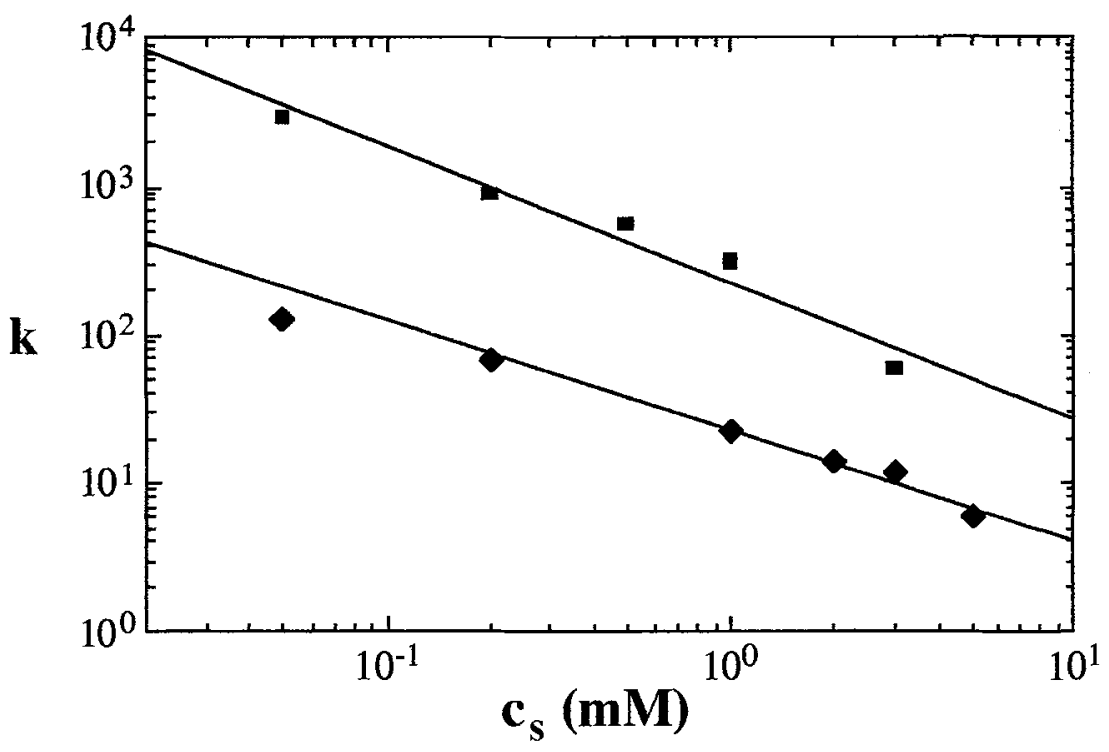

Fig. 5. The slope $\mathrm{k}$ plotted as a function of the salt concentration: ( $)$, PFA spherical latex; $(\mathbf{D})$, PTFE rod-like latex.

Since no theory of the Kerr effect in presence of interparticle interactions is, to our knowledge, presently available, we have developed an ad hoc phenomenological model which is based on a mean field argument.

We start from the general expression of the specific Kerr constant for a dispersion of independent particles occupying the volume fraction $\Phi[26,27]$ : 


$$
\underset{\Phi}{\mathrm{B}}=\frac{\left(\mathrm{n}_{\mathrm{s}}^{2}+2\right)^{2}}{270 \mathrm{~V} \lambda \mathrm{n}_{\mathrm{S}} \mathrm{E}_{\mathrm{o}} \mathrm{k}_{\mathrm{B}} \mathrm{T}}\left(\alpha_{1}^{o}-\alpha_{2}^{o}\right)\left(\alpha_{1}^{\mathrm{e}}-\alpha_{2}^{\mathrm{e}}\right)
$$

where $\alpha_{1}^{o}$ and $\alpha_{1}^{\mathrm{e}}$ are, respectively, the optical and electrical polarizability of the particle along the symmetry axis, $\alpha_{2}^{\mathrm{o}}$ and $\alpha_{2}^{\mathrm{e}}$ are the polarizabilities perpendicularly to the symmetry axis, $\mathrm{V}$ is the particle volume, $\varepsilon_{0}$ is the vacuum dielectric constant, and $n_{s}$ is the index of refraction of the solvent. Eq. 1 indicates that the existence of a nonzero Kerr constant implies the simultaneous presence in the particle of an electrical anisotropy (which produces the orientation of the particle when the electric field is applied) and of an optical anisotropy (which allows to detect the orientation of the particle by optical means). We observe that, in our case, the anisotropy of the electrical polarizability is essentially determined by the form of the particle and by the difference in dielectric constant with the solvent, while the optical part is dominated by the intrinsic anisotropy coming from the partially crystalline internal structure of the particles. This allows to simplify the general expressions for the anisotropy of polarizabilities (given in Eqs. 2 and 3 of Ref.10), so to obtain the approximate formulas:

$$
\begin{aligned}
& \left(\alpha_{1}^{\mathrm{e}}-\alpha_{2}^{\mathrm{e}}\right)=\mathrm{V} \varepsilon_{\mathrm{o}} \varepsilon_{\mathrm{s}} \frac{\left(\mathrm{L}_{2}-\mathrm{L}_{1}\right)\left(\varepsilon_{\mathrm{p}}-\varepsilon_{\mathrm{s}}\right)^{2} / \varepsilon_{\mathrm{s}}^{2}}{\left[1+\mathrm{L}_{1}\left(\varepsilon_{\mathrm{p}}-\varepsilon_{\mathrm{s}}\right) / \varepsilon_{\mathrm{s}}\right]\left[1+\mathrm{L}_{2}\left(\varepsilon_{\mathrm{p}}-\varepsilon_{\mathrm{s}}\right) / \varepsilon_{\mathrm{s}}\right]} \\
& \left(\alpha_{1}^{o}-\alpha_{2}^{o}\right)=V \varepsilon_{o} n^{2} \frac{\left(n^{2} 1^{-} n^{2}\right) / n^{2} s}{\left[1+\left(n^{2} 1^{2} n^{2} s\right) /\left(3 n^{2}\right)\right]\left[1+\left(n^{2} 2-n^{2}\right) /\left(3 n^{2}\right)\right]}
\end{aligned}
$$

where $\varepsilon_{\mathrm{S}}$ is the relative dielectric constant of the solvent, $\varepsilon_{\mathrm{p}}$ is the average relative dielectric constant of the particles, $\mathrm{L}_{1}, \mathrm{~L}_{2}$ are form factors which depend only on the particle geometry [30]. We recall that $\mathrm{L}_{1}$ $+2 \mathrm{~L}_{2}=1$.

It should be noted that if there were no correlation between the direction of the geometrical axis and that of the optical axis of the particle, no electric birefringence could be expected. Indeed, averaging over all the particles would correspond to summing equal amounts of positive and negative birefringence contributions. So we assume that the two directions coincide (to account for the positive sign of the birefringence): this is reasonable if one considers that the direction of the optical axis coincides with the average direction of crystal growth, so that one may expect the particles to be slightly elongated along this direction.

Considering that the particle shape is only slightly different from spherical and that $\varepsilon_{p}$ is much smaller than $\varepsilon_{\mathrm{s}}$, Eq.2 can be further approximated as:

$$
\left(\alpha_{1}^{\mathrm{e}}-\alpha_{2}^{\mathrm{e}}\right) \approx-\alpha_{\mathrm{p}} \frac{3 \mathrm{f}^{2}}{10} \approx \mathrm{V} \varepsilon_{\mathrm{o}} \varepsilon_{\mathrm{s}} \frac{9 \mathrm{f}^{2}}{20}
$$


where $\alpha_{\mathrm{p}}=\mathrm{V} \varepsilon_{\mathrm{o}}\left(\varepsilon_{\mathrm{p}}-\varepsilon_{\mathrm{s}}\right)\left[3 \varepsilon_{\mathrm{s}} /\left(\varepsilon_{\mathrm{p}}+2 \varepsilon_{\mathrm{s}}\right)\right] \approx-1.5 \mathrm{~V} \varepsilon_{\mathrm{o}} \varepsilon_{\mathrm{s}}$ is the particle excess polarizability with respect to the solvent, and $\mathrm{f}^{2}=1-(\mathrm{b} / \mathrm{a})^{2}$.

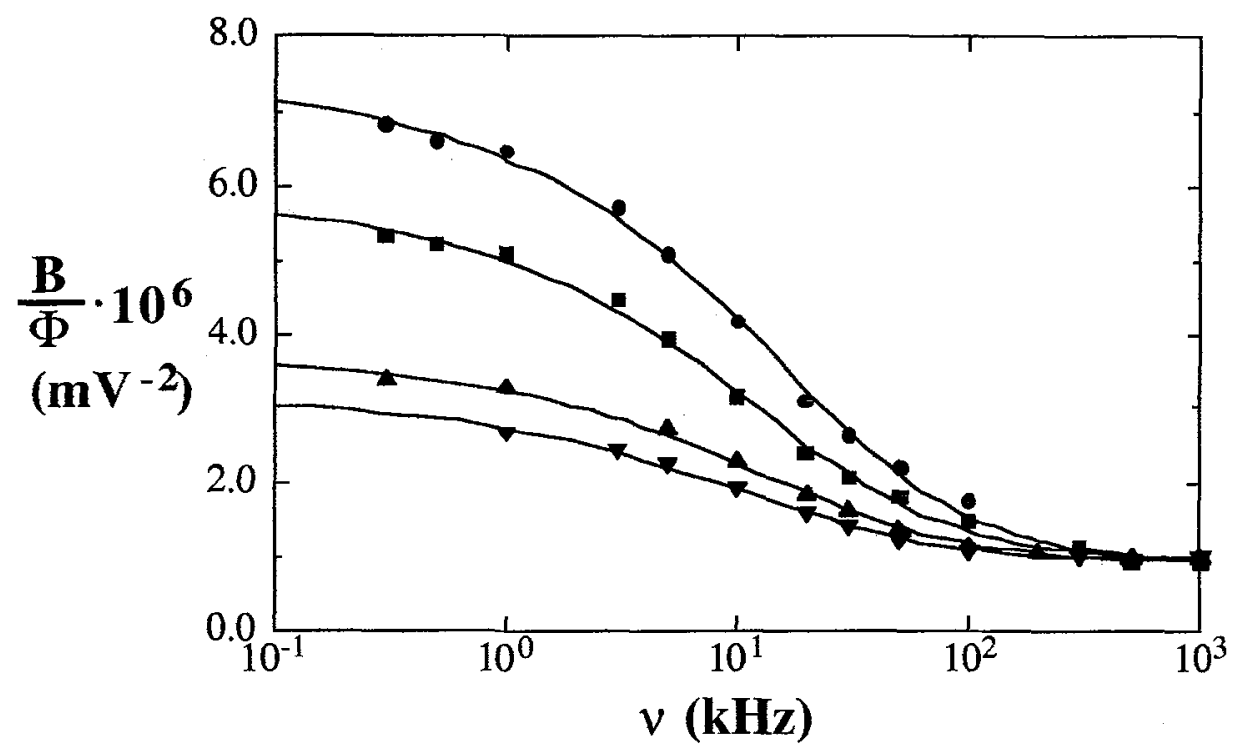

Fig. 6. The specific Kerr constant measured as a function of the frequency of the electric field, for the PTFE latex with no added salt, at four distinct volume fractions: $(\boldsymbol{\nabla}), \Phi=0.04 \% ;(\boldsymbol{\Delta}), \Phi=$ $0.09 \% ;(\square), \Phi=0.3 \% ;(C), \Phi=0.5 \%$; . The full lines represent a fit with Eq.6.

The data reported in Figs.2-4 show that, besides the effects coming from the intrinsic properties of the particles and from charge fluctuations, there is an additional contribution to the Kerr constant of the dispersion which presents the following features: i) it contains a term proportional to $\Phi^{2}$ whereas singleparticle effects are proportional to $\Phi$, ii) it is strongly dependent on the concentration of added salt, iii) it is a decreasing function of the frequency of the applied field. The first two features clearly indicate that the effect depends on the overlap of the counterion clouds surrounding each particle: when the overlap is reduced, either by increasing the ionic strength or by decreasing the particle concentration, the effect disappears. We have already reported a similar observation from a TEB experiment on dispersions of rod-like polytetrafluoroethylene latex particles [9]. Since the limit value of $B / \Phi$ at low frequency for vanishing volume fraction is almost coincident with the high frequency value, we have to conclude that undissociated counterions give a little contribution to the polarizability in the single particle limit.

We propose a mean field approach to interparticle interactions [10]: if the effect of.interactions is that of modifying the local field that a particle effectively feels, we can tentatively assume that the specific 
Kerr constant can still be calculated with Eqs.1-3 by simply inserting, instead of the relative dielectric constant of the solvent, the real part of the relative dielectric constant of the solution, $\varepsilon_{\text {sol }}$. If we use for the electrical anisotropy the approximated expression given by Eq.4, we find that $B / \Phi$ is proportional to the relative dielectric constant of the solvent. Therefore, the proposed mean-field approach implies that, for our system, $\mathrm{B} / \Phi$ should be approximately proportional to $\varepsilon_{\mathrm{sol}}$.

Several theoretical papers [21-23,31,32] have been devoted to the calculation of $\varepsilon_{\mathrm{sol}}$ for spherical macroions. Ref.31 presents numerical results which cover a wide range of values of the ratio $R / l_{D H}$, where $\mathrm{l}_{\mathrm{DH}}$ is Debye-Hückel length, whereas Refs.21-23 give analytical results valid, however, only for large values of $R / l_{\mathrm{DH}}$. We base our discussion mainly on the work by Chew and Sen [22]. At low volume fractions, $\varepsilon_{\text {sol }}$ can be written as [21-23]:

$$
\varepsilon_{\text {sol }}=\varepsilon_{\mathrm{s}}[1+\mathrm{k} \Phi \mathrm{F}(\omega)]
$$

where $\mathrm{k}$ depends on the particle properties and on the ionic strength of the solution, and $F(\omega)$ is given by:

$$
F(\omega)=\frac{1}{\left(1+\omega^{1 / 2} \tau_{s}^{1 / 2}\right)\left(1+\omega \tau_{s}\right)}
$$

where $\omega=2 \pi v$ and $\tau_{\mathrm{s}}=\mathrm{R}^{2} /(2 \mathrm{D})$ is a relaxation time, $\mathrm{D}$ being the diffusion coefficient of the dissociated counterions.

Since $B / \Phi$ is proportional to $\varepsilon_{\text {sol }}$, Eqs.5 and 6 can explain the observed linear dependence of $B / \Phi$ on $\Phi$ (see Fig.4), and the markedly non-Lorentzian frequency dependence of the specific Kerr constant. As shown by the full curves in Figs. 2 and 3 , the function $F(\omega)$ fits very well the experimental data.The obtained best-fit value is: $\tau_{\mathrm{s}}=(2.0 \pm 0.4) \mu \mathrm{s}$. Taking $\mathrm{D}=1.55 \times 10^{-5} \mathrm{~cm}^{2} / \mathrm{s}$ (this is the value measured at $0.01 \mathrm{M} \mathrm{NaCl}$ at $25^{\circ} \mathrm{C}$ ), the predicted $\tau_{\mathrm{S}}$ is $2.6 \mu \mathrm{s}$, roughly in agreement with the best-fit value. Since $\mathrm{D}$ changes somewhat with temperature and salt concentration, it is possible that the agreement can even be improved by performing the TEB experiment at a controlled temperature and by using a $\mathrm{D}$ value measured at the appropriate $\mathrm{c}_{\mathrm{s}}$.

Our data indicate that both the shape of the dispersion curve and the value of $\tau_{\mathrm{s}}$ are not significantly influenced by changes of $c_{\mathrm{s}}$ and $\Phi$ in the investigated range of parameters, whereas the value of $k$ is strongly dependent on $\mathrm{c}_{\mathbf{s}}$.

We have analyzed, according to Eqs.5 and 6, also the electric birefringence results we have obtained some years ago on dispersions of PTFE rods [9]. Some data are reported in Fig.6. The full lines represent the best-fit with the $F(\omega)$ given by Eq.6. The agreement is excellent. The best-fit value of the relaxation time is: $\tau_{\mathrm{s}}=(5.6 \pm 0.8) \mu \mathrm{s}$.

Lim and Franses [4] have fitted the observed frequency dispersion with a Cole-Cole function which contains two free parameters, the relaxation time $\tau_{\mathrm{s}}{ }^{\prime}$ and the exponent $\alpha$. Other semi-empirical functions 
were used by other authors [14]. We have made a comparison between the function given by Eq. 6 and a Cole-Cole function: $\mathrm{F}(\omega)$ can be very well approximated by a Cole-Cole function with $\tau_{\mathrm{s}}{ }^{\prime}=4 \tau_{\mathrm{s}}$, and $\alpha=$ 0.35 . However, we believe that the fit with Eq.6 is more significant because $F(\omega)$ comes from a theoretical model, and also because $F(\omega)$ contains only one free parameter.

By using eq.7.4 of Ref.22 we have derived the following approximate expression for $\mathrm{k}$ :

$$
\mathrm{k}=\frac{9}{8} \Phi \frac{\mathrm{Z}^{2} \mathrm{e}^{2}}{16 \pi^{2} \mathrm{R}^{4} \mathrm{k}_{\mathrm{B}} \mathrm{TN}_{\mathrm{A}} \mathrm{c}_{\mathrm{S}}}
$$

where $\mathrm{Z}$ is the charge of the particle in electronic units, and $\mathrm{N}_{\mathrm{A}}$ is Avogadro's number. A similar expression is given also in Ref.23. Although this point is not discussed in the theoretical papers, it seems plausible that one should take for $Z$ the value of the effective charge, that is, the bare charge minus that of the condensed counterions. It is therefore possible that $\mathrm{Z}$ is somewhat dependent on $\mathrm{c}_{\mathrm{s}}$. At fixed $\mathrm{R}$ and $\mathrm{Z}$, the theory of Chew and Sen predicts therefore that $\mathrm{k} \approx \mathrm{c}_{\mathrm{s}}{ }^{-1}$. Such a result is consistent with the data on PTFE particles. The agreement is less satisfactory for the PFA particles which are smaller. It should however be recalled that Eqs.5-7 are derived under the approximation $1_{\mathrm{DH}} / \mathrm{R} \ll 1$. Since, at $\mathrm{c}_{\mathrm{s}}=0.2$ $\mathrm{mM}, \mathrm{l}_{\mathrm{DH}}$ is about $22 \mathrm{~nm}$, it is possible that the data taken with the PFA particles at the lowest ionic strength cannot fully be interpreted by using Eq.7.

Concerning dispersions of polystyrene latex spheres, the available experimental data about the dependence of $\mathrm{k}$ on $\mathrm{c}_{\mathrm{s}}$ are rather confusing: Schwan et al [2] find that $\mathrm{k}$ is a decreasing function of the ionic strength, but subsequent investigations [3-6] show the opposite behavior.

Delacey and White [31] present numerical solutions for the dielectric increment in the range $1 \leq \mathrm{R} / 1_{\mathrm{DH}}$ $\leq 50$. They find that, at fixed electric potential on the slipping plane, $\zeta$, and at fixed $R, k$ is an increasing function of $c_{s}$. Since, at fixed $\zeta$, an increase of $c_{s}$ produces an increase of $Z$, the main problem for the comparison between experiment and theory seems to be the following: a change of the ionic strength is associated by a change of the effective charge or by a variation of the $\zeta$ potential? Generally speaking, it is possible, of course, that both quantities are dependent on $c_{s}$. In the case of the fluorinated polymer latices discussed in this paper, the observed behavior of $k$ versus $c_{s}$ can be explained by assuming a constant effective charge, whereas the behavior reported in Refs.3-6 is mainly consistent with the hypothesis of constant $\zeta$ potential.

\section{Conclusions.}

We have shown in this article that fluorinated latex (PFA) particles represent a very interesting model system for TEB studies of the dielectric properties of polyelectrolytes. The specific Kerr constant of the particle dispersion is rather large because the particles possess both an intrinsic optical anisotropy and a slightly elliptical average shape. 
The specific Kerr constant presents a contribution whose amplitude increases with the particle concentration and decreases with salt concentration. The frequency dispersion of such contribution is markedly non-Lorentzian, and fits very well the predicted frequency dispersion for the dielectric constant of the solution. A simple mean field argument is used to justify this latter result.

\section{Acknowledgements.}

We thank D. Lenti for the gift of the Hyflon latex particles, and C. Cametti for useful discussions. Parts of this work were written during a stay of one of the authors (V.D.) at the Aspen Center for Physics, Aspen, Colorado, U.S.A.. We acknowledge financial support from MURST 40\%.

\section{References.}

1. Mandel,M, and Odijk, T, Ann. Rev. Phys. Chem. 35 (1984) 75.

2. Schwan, HP, Schwarz, G, Maczuk,J, and Pauly,H, J. Phys. Chem. 66 (1962) 2626.

3. Springer,MM, Korteweg,A, and Lyklema,J, J. Electroanal. Chem. 135 (1983) 55; Lyklema,J, Springer,MM, Shilov, VN, and Dukhin, SS, J. Electroanal. Chem. 198 (1986) 19.

4. Lim,K-H, and Franses,EI, J. Colloid Interface Sci. 110 (1986) 201.

5. Myers,DF, and Saville,DA, J. Colloid Interface Sci. 131 (1989) 448, 461.

6. Ballario,C, Bonincontro,A, and Cametti,C, J. Colloid Interface Sci. 72 (1979) 304; 54 (1976) 415;

Bordi F, and Cametti C, Ber. Bunsenges. Phys. Chem. 95 (1991) 675.

7. O'Konsky,CT, and Haltner,AJ, J.Am.Chem.Soc. 78 (1956) 3604; Newman,J, and Swinney,HL, Biopolymers 15 (1976) 301.

8. Thurston,GB, and Bowling,DI, J. Colloid Interface Sci. 30 (1969) 34.

9. Bellini,T, Piazza,R, Sozzi,C, and Degiorgio,V, Europhysics Letters 7 (1988) 561.

10. Mantegazza,F, Giardini,M, Piazza,R, and Degiorgio,V, J.Phys.Cond. Matter 4 (1992) 8683.

11. Elias,JG, and Eden,D, Macromolecules 14 (1981) 410; Hans,M, and Bernengo,JC, Biopolymers 12 (1973) 2151.

12. Wijmenga,SS, and Mandel,M, J. Chem. Soc. Faraday Trans.1 84 (1988) 2483.

13. Tricot,M, and Houssier,C, Macromolecules 15 (1982) 854.

14. Ookubo,N, Teraoka,I, and Hayakawa,R, Ferroelectrics 86 (1988) 19.

15. Ookubo, N, Hirai,Y, Ito,K, and Hayakawa,R, Macromolecules 22 (1989) 1359.

16. Degiorgio,V, Bellini,T, Piazza,R, Mantegazza F. and Goldstein,RE, Phys. Rev. Lett. 64 (1990) 1043.

17. Krämer,U, and Hoffmann,H, Macromolecules 24 (1991) 256.

18. Hoffmann,H, Krämer,U, Thurn,H, J. Phys. Chem. 94 (1990) 2027.

19. Bellini,T, Mantegazza,F, Piazza,R, and Degiorgio,V, Europhys. Letters 10 (1989) 499. 
20. Piazza,R, Stavans,J, Bellini,T, Lenti,D, Visca,M, and Degiorgio,V, Progr. Colloid Polymer Sci. 81 (1990) 89; Piazza,R, and Degiorgio,V, Optics Commun. 92 (1992) 45.

21. Fixman,M, J.Chem.Phys. 72 (1980) 5177.

22. Chew,WC, and Sen,PN, J. Chem. Phys. 77 (1982) 4683.

23. Vogel,E, and Pauly,H, J. Chem. Phys. 89 (1988) 3823.

24. Kirkwood,JG, and Shumaker,JB, Proc.Natl.Acad.Sci.U.S. 38 (1952) 855.

25. Piazza,R, Degiorgio,V, and Bellini,T, J. Opt. Soc. Am. B 3 (1986) 1642.

26. Fredericq,E, and Houssier,C, "Electric Dichroism and Electric Birefringence" Clarendon (Oxford, 1973).

27. O'Konski,CT, Ed., "Molecular Electro-Optics" Marcel Dekker (New York 1976).

28. Jennings,BR, Ed., "Electro-Optics and Dielectrics of Macromolecules and Colloids", Plenum Press (New York, 1979).

29. Stoylov,SP, "Colloid Electro-Optics: Theory, Techniques, Applications" (Academic Press, London, 1991).

30. van de Hulst,HC, "Light Scattering by Small Particles" (Dover, New York, 1981).

31. DeLacey,EHB, and White,LR, J. Chem. Soc. Faraday Trans.2, 77 (1981) 2007.

32. Lyklema,J, Dukhin, SS, and Shilov, VN, J. Electroanal. Chem. 143 (1983) 1. 